\title{
Commissioning of the ATLAS Jet and Missing Energy Triggers with Beam collisions at the LHC
}

\author{
L. Zhao, New York University, USA, P.-H. Beauchemin, University of Oxford, UK, \\ on behalf of the ATLAS Collaboration
}

\begin{abstract}
We present the algorithms constituting the ATLAS jet and Missing Transverse Energy (MET) triggers and summarize the performance studies that have been made for these triggers with the $7 \mathrm{TeV}$ early LHC collision data. The strong online to offline correlation observed for the jet and MET quantities, as well as the good data to Monte Carlo (MC) agreement observed in the various figures of merit investigated (efficiency, rate, etc), demonstrate that these triggers perform at the level of our expectations.
\end{abstract}

\section{INTRODUCTION}

W ITH the $7 \mathrm{TeV}$ collision data collected recently, performance studies of the ATLAS trigger system are critical for a successful physics program. In particular, a large spectrum of physics results will rely on the capacity of the TDAQ system to select events based on the jets and Missing Transverse Energy. The jet triggers are not only mandatory for QCD studies, but also crucial for new physics discoveries, such as super-symmetric Higgs bosons and high-mass dijet resonances. The MET triggers would be the primary ones used in searches for processes involving new weakly interacting particles that could account for the dark matter indicated by astronomical observations. In addition, the MET triggers can also be used in combination with other triggers to control the rate of signatures involving low energy objects, like the measurement of non-boosted $\mathrm{W}$ bosons decaying in the tau channel. Finally, these triggers can be used for a large range of performance studies. For example, because of its negligible correlation with them, the MET trigger is ideal to estimate the efficiency of electron triggers.

The ATLAS experiment uses a three-level trigger system. The Level 1 (L1) is an electronics and firmware trigger, with a designed output rate of $75 \mathrm{kHz}$ and a typical operation rate of $20 \mathrm{kHz}$ during the data-taking period of this study. The Level 2 (L2) and Event Filter (EF) are software algorithms which together are called the High Level Trigger (HLT). As is the case for L1, L2 only processes the Regions of Interest (ROIs), has a designed rate of $3 \mathrm{kHz}$ and a typical rate of 4 $\mathrm{kHz}$. The EF trigger can access the full detector with more sophisticated software algorithms, has a designed rate of 200 $\mathrm{Hz}$ and a typical rate of $350 \mathrm{~Hz}$.

Manuscript received on November 16, 2010.

Long Zhao, is with New York University, New York, NY 10003 USA. (e-mail: long.zhao@nyu.edu).

H.-P. Beauchemin, is with University of Oxford, Oxford, Ox1 3RH UK (e-mail: h.beauchemin1@physics.ox.ac.uk).
For the jet triggers, L1 constructs jet elements from $2 \times 2$ sums of the approximately 7200 calorimeter trigger towers (TTs). Then it uses a sliding-window algorithm to cluster jet elements to form jets. The eta-phi position of the Regions of Interest (ROIs) of the L1 jets is passed to the HLT after an L1 trigger accept. The $\mathrm{L} 2$ trigger is based on a simplified version of the offline cone clustering algorithm, limited to a maximum of three iterations on calorimeter clusters around the ROIs with full granularity. The EF uses the same reconstruction algorithms as the offline reconstruction, but it uses different calorimeter calibration due to the fact that the jets formed at EF level only use clusters inside the ROIs surrounding the direction of the L1 jets.

For the MET triggers, the L1 uses a look-up table to accept or reject events based on Ex and Ey, which are the vector sum of the x-component and y-component of each TTs energy correspondingly. These quantities are computed in the jet energy processing together with the L1 jets and L1 SumEt. At L2, the MET triggers add muon information, which is absent at L1. At EF, the MET triggers can access the full calorimeter giving the most precise online MET measurements. Since muons do not contribute much to MET or SumEt for minbias events, below we do studies only of L1 and EF.

In this paper, the study of the jet triggers is based on data taken in the period from the beginning of April to the beginning of June 2010 [1], and the study of the MET triggers is based on data taken in period from August 19 to August 29 2010.

\section{Performance Studies of Trigger measurements}

Both the jet and MET quantities are reconstructed online at all three levels of trigger. Thus, it is important to compare these online measurements with their corresponding offline measurements, as well as to compare data to MC simulation.

\section{A. The Jet Angular and Transverse Momentum Measurement}

Fig. 1 shows that the results of the jet angular measurement for data are in agreement with those for the MC simulation. Also, we can see that the ROIs of the jet trigger point closely, to within about 0.3 angular distance ( $\phi$ or $\eta$ ) to the offline jet positions. Fig. 2 shows the ratio of L2 ET to offline anti-kt algorithm jet ET versus offline ET and versus $\eta$. The simulation and data agree well. A small discrepancy between data and MC in the high-eta region is known to be caused by 

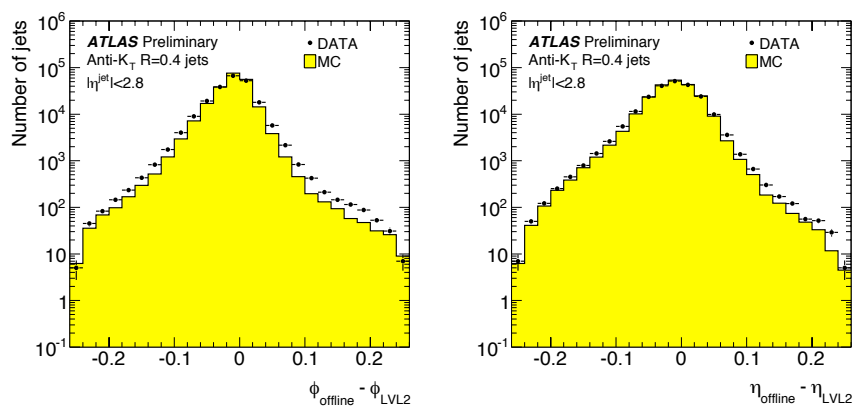

Fig. 1. Difference in $\phi$ (left) and $\eta$ (right) values between jets reconstructed at Level-2 to the offline anti-kt algorithm value with clustering parameter $\mathrm{R}=0.4$. Trigger and offline jets are matched for comparison by selecting the smallest $\Delta R$ separation.
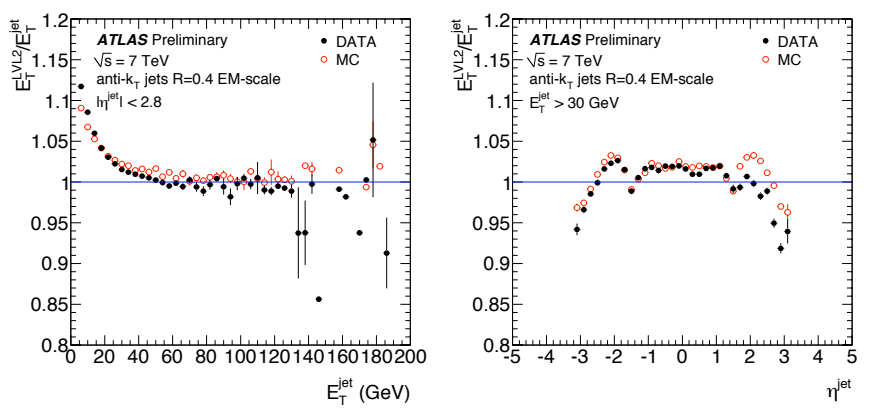

Fig. 2. Ratios of transverse momentum of jets reconstructed at L2 and with the offline anti-kt algorithm with clustering parameter $\mathrm{R}=0.4$, as a function of the ET (left) and $\eta$ (right) of the offline jet. At both levels, the jets are calibrated to the electromagnetic scale.
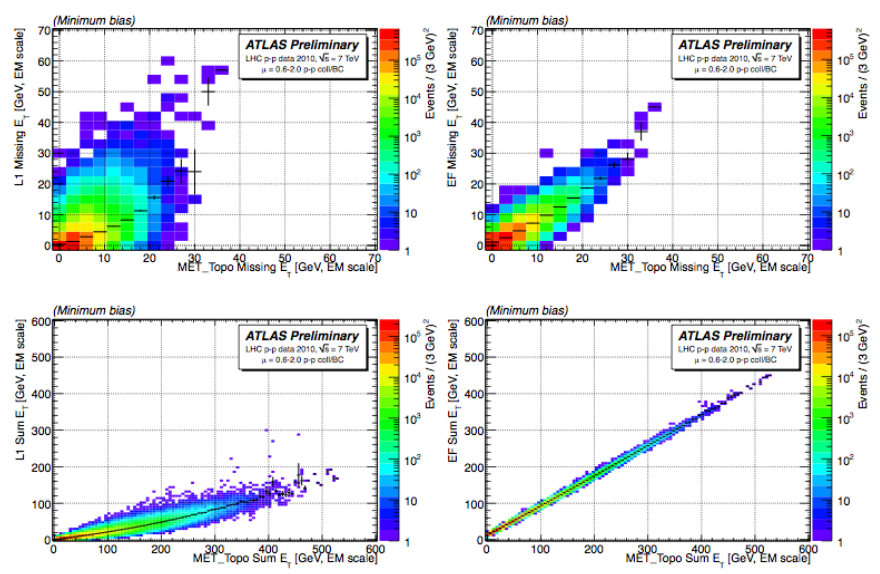

Fig. 3. Correlation of L1 Missing ET (top left), EF Missing ET (top right), L1 Sum ET (bottom left) and EF Sum ET (bottom right) with the reference offline algorithm (MET_Topo), for all events triggered by the minimum bias scintillators (energy at EM scale for both trigger and offline quantities). MET_Topo loops over all topological clusters, obtained from seed cells with energy above 4 noise RMS, adding the neighboring cells above 2 RMS, and finally taking all adjacent cells. The average number of collisions per bunch crossing ranged from 0.6 to 2 in the sample (Aug 2010).

a high voltage test occurring in the forward part of calorimeter during the period examined here [1].
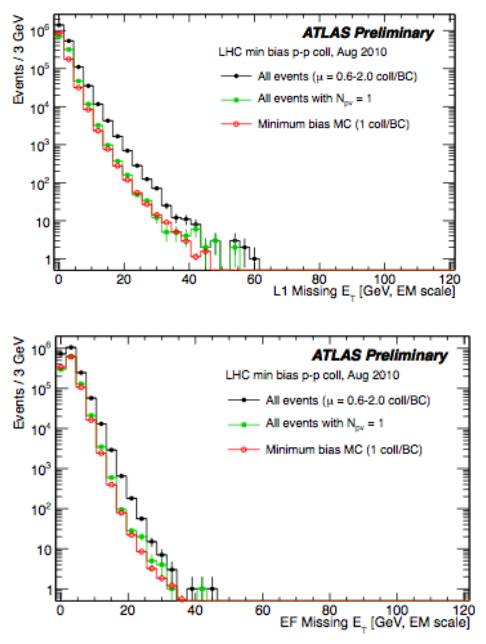

Fig. 4. L1 (top) and EF (bottom) Missing ET measured for data and simulated for minimum bias events for all events and for the subset obtained by rejecting events with multiple reconstructed primary vertices. Energy is at EM scale. The simulation (normalized to the integral) reproduces the spectrum well when a single interaction is considered.

\section{B. The Correlation between Trigger and Offline MET}

The plots shown in Fig. 3 indicate strong correlation between online Missing ET and offline measurements, both for Missing ET and Sum ET. The superimposed profile histogram (black crosses) shows that the energy scale at L1 is different from the EF (or offline) EM-scale. Also, it is clear that EF has better correlations with offline than L1 for both Missing ET and Sum ET. This is because a very conservative L1 noise suppression scheme was adopted in the 2010 ATLAS runs, making the L1 triggers more sensitive to localized objects such as jets than globally distributed energy, like Missing ET or Sum ET.

\section{Comparison of MET Trigger on Data and Simulation}

As the MC only simulates one primary vertex while there may be multiple primary vertices in data, we can see in Fig. 4 a clear pile-up impact on measured Missing ET. After cutting on the number of reconstructed primary vertices, data has much better agreement with simulation. By comparing data to simulation, we are confident that the MET trigger measurements are well understood.

\section{TRIGger EfFicIENCY STUdies}

Studying the trigger efficiency is another crucial task for understanding the trigger performance. Trigger efficiency is calculated as the ratio of the trigger quantity distribution such as jets or MET in events that passed the trigger with respect to a reference distribution. The reference distribution should be unbiased as much as possible. We choose the minimal bias events selected by the trigger MBTS_1 in this study.

\section{A. The Jet Trigger Efficiency}

In Fig. 5, we show the L1 jet trigger efficiency as a function of offline jet $p_{\mathrm{T}}$. There is good agreement between data and 

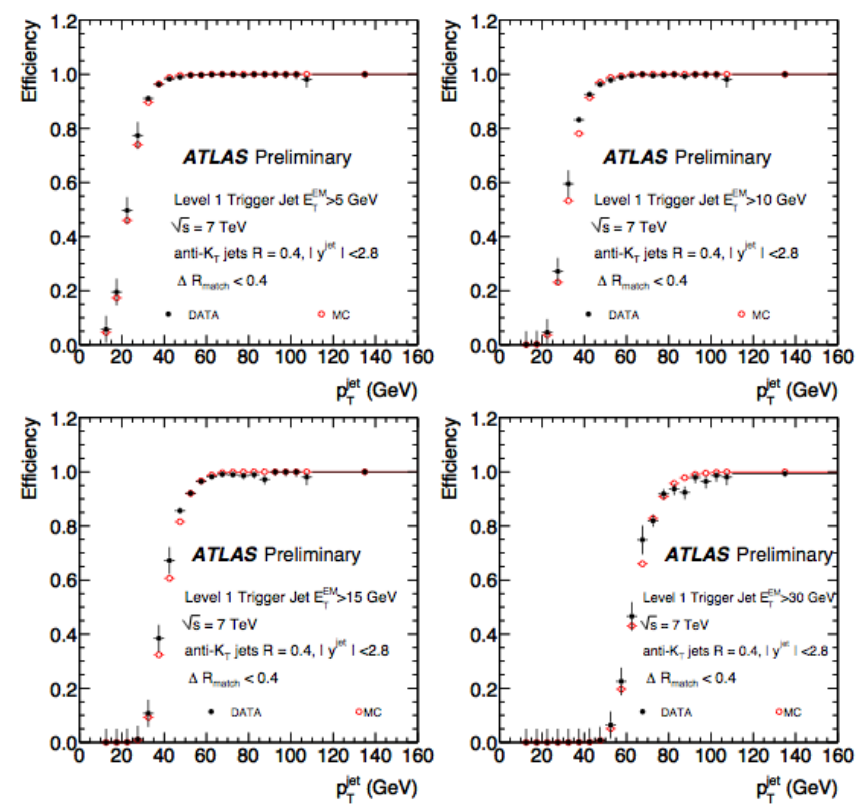

Fig. 5. The efficiency for an anti-kt jet with $\mathrm{R}=0.4$ to satisfy $\mathrm{L} 1$ as a function of the jet $p_{\mathrm{T}}$, integrating over $|y|<2.8$. Left to right and top to bottom are efficiencies for four lowest L1 thresholds: 5, 10, 15 and $30 \mathrm{GeV}$.

simulation, although the details differ. The slight inefficiencies at the high $p_{\mathrm{T}}$ plateau region are due to offline jets being split into two by the L1 jet algorithm. This is because the size of L1 jets is smaller than the typical offline jets. Therefore, potential inefficiencies may happen above the plateau region.

\section{B. The $\eta$ dependence of Jet Trigger Efficiency}

In Fig. 6, the efficiency for the lowest L1 trigger threshold is shown as a function of jet $\eta$ for three different regions of the offline reconstructed jet transverse momentum $\left(20<p_{\mathrm{T}}<40\right.$ $\mathrm{GeV}$ at the top; $40<p_{\mathrm{T}}<60 \mathrm{GeV}$ and $p_{\mathrm{T}}>60 \mathrm{GeV}$ at the bottom), and for two values of $\mathrm{R}$ ( 0.6 for the top left; 0.4 for the others), as used in the offline jet reconstruction. The low transverse momentum region (the top two plots) exhibits a strong dependence on $\eta$, particularly in the regions around $|\eta| \sim 1.5$ the region of transition from the barrel and the endcap calorimeters [1]. In general, data and simulation are in good agreement. There is better agreement for jets reconstructed with radius $\mathrm{R}=0.6$ (the up left plot) than $\mathrm{R}=0.4$ (the up right plot). This indicates the impact of jet size on jet trigger efficiency.

\section{The MET Trigger Efficiency}

In Fig. 7, the "turn-on" curves of EF-only Missing ET triggers with lowest thresholds are ploted. The efficiencies determined from data and $\mathrm{MC}$ agree well, indicating that MET HLT algorithms are performing as expected. Also, the sharp efficiency increase demonstrates that the distortion of the offline quantities by the MET trigger preselection are relatively small. Here, the EF-only Missing ET trigger means the trigger has a hypothetical cut at EF only. The plots showing EF-only Missing ET performance were made by means of a special trigger chain without L1 preselection.
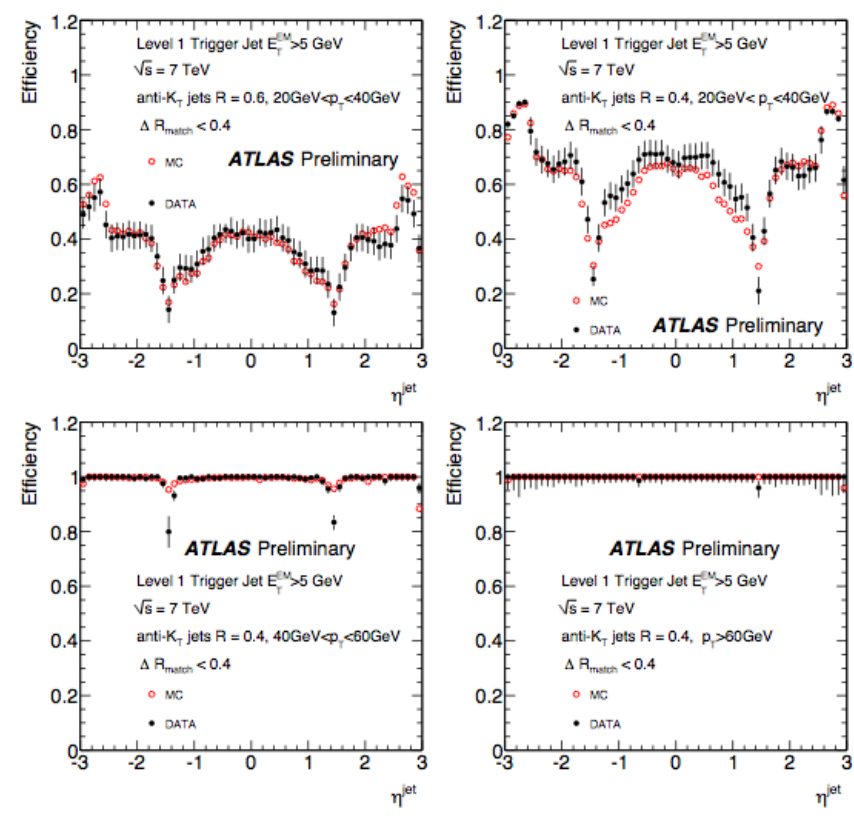

Fig. 6. The efficiency for an anti-kt jet to satisfy L1 as a function of the jet $\eta$ for the $5 \mathrm{GeV} \mathrm{L1}$ jet threshold. The efficiency is integrated in three bins in $p_{\mathrm{T}}: 20<p_{\mathrm{T}}<40,40<p_{\mathrm{T}}<60$, and $p_{\mathrm{T}}>60 \mathrm{GeV}$ which correspond to the three regions of the efficiency as a function of jet $p_{\mathrm{T}}$. The upper-right anlower plots are for jets reconstructed ofine with $\mathrm{R}=0.4$; the upper-left plot requires $\mathrm{R}=0.6$.
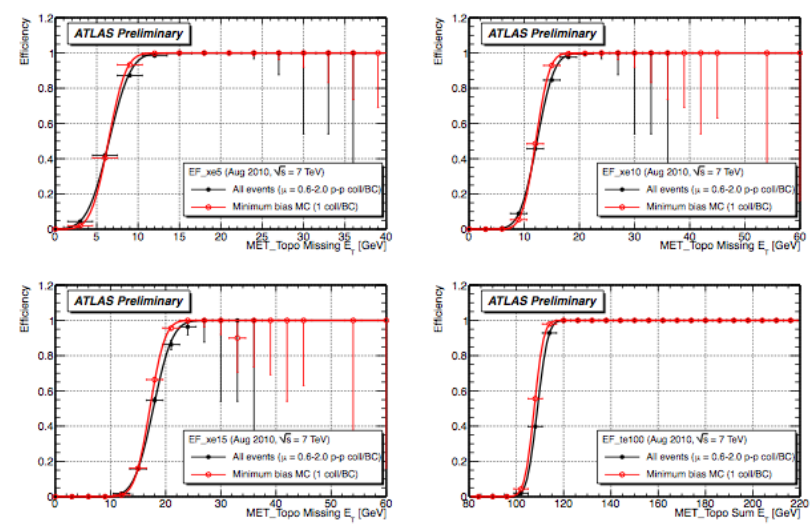

Fig. 7. The efficiency of EF-only Missing ET thresholds at 5, 10, $15 \mathrm{GeV}$ and (bottom-right) of a Sum ET threshold at $100 \mathrm{GeV}$, as function of the reference (MET_Topo) quantities. MET_Topo loops over all the topological clusters obtained from seed cells with EM-scale energy above 4 noise RMS, adding the neighboring cells above 2 RMS and all adjacent cells.

\section{ACKNOWLEDGMENT}

We'd like to thank Allen Mincer and Diego Casadei for their help in preparing this paper.

\section{REFERENCES}

[1] The ATLAS Collaboration, Performance of the ATLAS Jet Trigger in the Early $\sqrt{s}=7 \mathrm{TeV}$ Data, ATLAS-CONF-2010-094, October 2010. 